\title{
A Survey of Manual Detorsion of The Testicle
}

\author{
Paddy Dewan ${ }^{1}$, Bhavish Kowlessur ${ }^{2}$ and John Lazarus ${ }^{2}$ \\ ${ }^{1}$ Kind cuts for Kids and Urology Unit, Red Cross Children's Hospital, Australia \\ ${ }^{2}$ University of Cape Town, South Africa
}

Submission: October 29, 2016; Published: November 14, 2016

*Corresponding author: Dewan P, Kind cuts for Kids and Urology Unit, Red Cross Children's Hospital Post Box 152, Parkville 3052, Australia, Email: paddy@paddydewan.com

\section{Abstract}

Purpose: A nine question survey was established on an internet survey provider, aimed to understand attitudes to two principle aspects of management of testicular torsion. Respondents were asked about their experience and attitude toward the use of preoperative manual detorsion, the analgesic usage for that manoeuvre and the approach to the significantly ischaemic testicle at the time of scrotal exploration.

Materials Methods: A monkey survey platform included 9 questions, the results of which were analysed via an access database. In all, 561 emails were sent from the authors, and the numbers linked via the Australasian Urology and Paediatric Surgery societies, for privacy reasons, were not defined. A total of 285 responses were collected; 181 via emails responses and 104 via a web link.

Results: Results of 285 who did respond, 134are Urologists, 33 Urology trainees, 45Paediatric Urologists,58Paediatric Surgeons and 15 Paediatric Surgery trainees.19 of 58 (32.8\%) of Paediatric surgeons, 32 of 45 (66.7\%) Paediatric Urologists and 84of 134 (62.7\%) of Urologists had untwisted a testicle before theatre, but 12 (25.0\%) of 48 trainees have either never been taught or do not believe in the manual detorsion; $46.7 \%$ of those in Paediatric Surgery compared to four of 33 urology trainees $15.1 \%$.

Conclusion: The majority of surgeons rely on manual detorsion of the testicle as part of the management of patients with a twisted testicle in the adolescent age group, and there appear to clinical criteria that are used to assess the appropriateness of the manoeuvre.

Keywords: testicular torsion; spermatic cord torsion; manual detorsion; capsulotomy; testicular infarction.

\section{Introduction}

Testicular torsion occurs in 1 in 4000 males before the age of 25 years [1], but despite the common occurrence and multiple papers published on the use of manual detorsion there appear to be strong views against the use of the technique, and a wide variation between the different specialties in how to manage testicular torsion patients. Certainly, there are not any public education programs to ensure teenage boys know how to self detort a twisted testicle, despite the high testicular loss rate from torsion [2-3] and the high incidence of litigation when testicular loss occurs.

How to manage the infracted and ischemic testicle like-wise seems opinionated, rather than based on science as highlighted in studies of the testis compartment syndrome [1-4], which is managed by incision by most and insertion of tunica vaginalis in recent studies [1-4].
There are a number of anaesthetic techniques advocated by respondents to the survey, but little literature on the subject [5-6]. And, many are sceptical about the need cause to cause severe pain when they optimistically reflect on easy access to theatre, a view that fails to appreciate that most patients attend a non-hospital practitioner prior to arriving in the emergency department, therefore it would be important for the community, and community health workers, to be aware of a procedure that would buy time. There are now many papers that advocate manual detorsion [5-12] including publications from the 19th Century [13].

A further barrier to the use of preoperative manual detorsion appears to be uncertainty about the direction of the twist. However, this does not appear to be a problem in the clinical setting, as it the instant pain relief is a marker of the direction in the early presentation cases. Several studies have documented 
the direction, with most authors noting medial rotation as part of the pathophysiology that is solved by laterally rotating the testicle in the majority of boys, but not all $[5,7,9]$.

To understand the range of views from those specialties that care of these young men, a brief survey was constructed to ascertain both the attitude and experience of manual detorsion from the different craft groups, namely, Paediatric Surgeons, Urologists and Paediatric Urologists.

\section{Materials Methods}

A monkey survey platform included 9 questions, the results of which were analysed via a Microsoft Access database. In addition to individual emails, emails to an international Paediatric Urology discussion group, the link was provided to the Urological Society of Australasia, members of the South African Urological Association, the Australasian Paediatric Surgery Society. In all, 561 emails were sent from the authors, and the numbers linked via the Australasian Urology and Paediatric Surgery societies, for privacy reasons, were not defined.

A total of 285 responses were collected; 181 via emails responses and 104 via a web link. Of those 533 contacted directly from the survey team 22 opted out, 23 emails bounced, 159 were unopened, and of the 329 were opened 181 were completed. The other 104 completed responses were via a web link provided to members of the Australasian Urology and Paediatric Surgery societies. The literature was reviewed through Medline and the service of the University of Cape Town and the Royal Australasian College of Surgeons.

\section{Results}

Results of 285 who did respond, 134 are Urologists, 33 Urology trainees, 45 Paediatric Urologists, 58 Paediatric Surgeons and 15 Paediatric Surgery trainees.167 trained surgeons were more than 10 years post-graduation, of whom 96 (57.5\%) had untwisted a testicle before theatre; 19 (11.4\%) had untwisted a testicle in many patients. 19 of $58(32.8 \%)$ of Paediatric surgeons, 32 of 45 (66.7\%) Paediatric Urologists and 84 of $134(62.7 \%)$ of Urologists had untwisted a testicle before theatre, which by country showed 19 of 26 (73\%) USA surgeons, 83 of $107(77.6 \%)$ of South African surgeons and only 23 of 74 (31\%) Australian surgeons have untwisted a testicle before theatre, but only one of 15 (6.7\%) Paediatric surgical trainees, most of them are Australian. Twelve (25.0\%) of 48 trainees have either never been taught or do not believe in the manual detorsion; $46.7 \%$ of those in Paediatric Surgery compared to four of 33 urology trainees $15.1 \%$ of the 126 of 285 who had not untwisted a testicle before theatre, 5 asserted that there was no waiting time to get into theatre, 24 of 285 (8.4\%) had never been taught and $47(16.5 \%)$ contended they do not believe it to be appropriate, which included 5 of those who have never been taught, one who had previously detorted testes off those who do use manual detorsion, 63 of the 159 (41.0\%) would not attempt manual preoperative detorsion if there was scrotal oedema, an additional $64(40.2 \%)$ regarded severe pain as an exclusion, 7 reported they would always try.

At the time of the manual detorsion 113 (71.1\%) do not use analgesia, 26 (16.4\%) use local and $22(13.8 \%)$ use nitrous oxide or intravenous agents, without local anaesthetic. At the time of exploration 159 (66.7\%) would be inclined to incise the tunica as a matter of course if considering retaining the testicle, 154 $(54.0 \%)$ would only remove the testicle if it were obviously dead or did not bleed with incision of the tunica. Six would never remove the testicle.

\section{Discussion}

Our study related to four aspects of the care of patients with intratunical testicular torsion; the use and attitude toward detorsion, the role of analgesia, the management of a testicle in a marginal state after detorsion, and the decision making related to, and the role of, orchidectomy. In 1893, Nash described the case of a 19 year old school boy with acute pain in the testis who has his pain relieved by manual detorsion 13 , being the first to state the dilemma about the direction to rotate to untwist the testicle. "I rotated the epididymis in front of the testicle to the patient's left, but it caused more pain and would not stay in that position. I next rotated it to the patient's right, so that it resumed its normal position behind the body of the testis. A series of three cases were then presented in 1898 [14].Since then it has become apparent that most testes rotate medial and untwisted laterally, but not all [5-7], with resolving the twist being by external rotation in the majority [14-15].

Those responding to the survey reported that they were concerned that it was not possible to identify that the twist has been completely resolved, and others suggested the procedure is too painful. Incomplete detorsion have been recorded but is uncommon, and with the proviso that the presentation is early, detorsion seems reliable and quick, so quick that most surgeons in the survey do not take extra "ischemic" time to enable provision of pain relief, but rely on the instant pain relief that occurs with the manual detorsion in those cases without scrotal redness and oedema. A number of authors have presented a number of analgesic alternatives, including regional blockade [5]. The responses in the survey included 36 practitioners who use local anaesthetic, 11 uses nitrous, 19 others use other agents including morphine.

The provocative question in our study related to the attitude toward manual detorsion; despite the number of studies indicating that the technique can be successful in selected cases, 10 surgeons argued that the availability of theatre precluded the use of the manual detorsion, while 47 stated they "did not believe in it", or had not been trained, particularly Australasian Paediatric Surgeons, noting however that the survey was not sent to all specialists in all geographical areas. Disappointingly, 25 
claimed to have "never been trained" in a technique that clearly has a good track record, and strong support of the majority of clinicians involved in care of these patients. Notable 5 of those who had never been trained "did not believe in it", which might reasonably be thought to lack an open minded approach to therapeutic options.

It is known that the two most important factors determining testicular damage are the time from the onset of symptoms to the reduction of torsion, and the degree of twisting in the cord. Experimental studies in dogs show the elimination of all spermatogenic and Sertoli cells by six hours of testicular ischaemia, and the elimination of Leydig cells by $10 \mathrm{~h}$ of ischemia [16]. Some degree of atrophy is almost inevitable after torsion of more than four hours duration. Infarction can occur as early as four hours if the cord has twisted through several revolutions. After 10 hours of torsion most patients will have significant atrophy, unless the torsion was limited to $180-360^{\circ}$ [17]. With torsion of more than $360^{\circ}$ lasting more than 24 hours all patients will have complete or severe atrophy [18].

Moreover, it is known that unilateral testicular torsion interferes with subsequent spermatogenesis. The sperm count and the degree of atrophy both correlate closely with the duration of torsion [17]. Patients who have atrophy or who have undergone orchidectomy have a significantly lower sperm count than those without atrophy [19]. Abnormalities noted in the contra lateral testis include extensive apoptosis in the germinal epithelium, atrophic Leydig cells, malformation of the late spermatids, fewer late spermatids, and pathological changes in Sertoli cells. Trauma to the blood-testis barrier initiated by torsion may induce the release of cytokines, which damage the contra lateral germinal epithelium [20].

While easy access to theatreallows for definitive management, the reality is that the mean time between presentation and intraoperative detorsion often varies between 1 to 7 hours, even in areas where healthcare is well developed, and best community care would result in teenage boys being aware of the diagnosis, using their mobile phone camera to document the pathology and untwisting the testicle themselves, alternatively the primary care physician doing likewise. The primary pathophysiological event in testicular torsion is ischemia of the testis followed by reperfusion after detorsion [21]. Thus, testicular torsion can be thought of as an ischemia/reperfusion injury to the testis.

Repair of testicular torsion is followed by a period of germ cell apoptosis, accumulation of testicular neutrophils and increased testicular oxidative stress. Testicular vascular E-selectin expression is increased after de-torsion as are a number of cytokines important in the recruitment of neutrophils [22]. The testis is covered by the tunica albuginea, a fairly strong and inelastic layer. As a result, tissue changes from ischemiareperfusion leads to oedema, increased intratesticular pressure and reduced perfusion pressure. Hence perpetuating testicular ischemia [23]. This has been termed the testicular compartment syndrome.

The latter part of the study questionnaire sort to understand surgical management of a testicle in a marginal state after detorsion, and the decision making related to, and the role of, orchidectomy. Traditional intraoperative management involved either detorsion and orchidopexy or orchidectomy depending on the subjective assessment of testicular viability by the surgeon. Recently a new method involving incision of the tunica albuginea and flap coverage with Tunica Vaginalis was described. This directly addresses the testicular compartment syndrome and may improve testicular salvage rates. 190 surgeons indicated that they would incise the tunica in an ischemic testicle, which has been investigated by at least two groups, who suggest the use of covering the exposed tissue with tunica vaginalis [1,4]. 85 surgeons do not incise the tunica, but others present argument for variable use of incision on the clinical scenario, which seems a rational, more guarded approach.

Interestingly, six surgeons would never remove an infarcted testicle, 124 would always remove the testicle, but not incise the testicle before doing so. The other 139 surgeons and trainee would incise the tunica first to check for viability before making the final decision to perform an orchidectomy. Clearly, more work needs to be done to understand the outcome for the dead testicle left behind, and the determinant of viability to help develop patient specific international guidelines-particularly focussed on the understanding that no two patients are the same.

\section{Conclusion}

The majority of surgeons rely on manual detorsion of the testicle as part of the management of patients with a twisted testicle in the adolescent age group, and there appear to be clinical criteria that are used to assess the appropriateness of the manoeuvre. However, it would also appear the technique is not as widely used as possible with a particular concern that trainees in both surgery and urology have either never been taught or "don't believe" in the technique felt appropriate by the majority.

Given the success of the technique as published since 1893, the ability of some patients to untwist their own torsion, it would seem improved community, general practitioner and speciality knowledge of the first aide for twisted testicles would be appropriate. What should be done when a torsion case gets to theatre, and how the degree of damage should be assessed at the necessary surgical intervention subsequently, obviously needs to be subjected to enhanced scientific rigor.

\section{References}

1. Kutikov A, CasaleP,White MA, Meyer WA, ChangA, etal.(2008) Testicular Compartment Syndrome: A New Approach to Conceptualizing and Managing Testicular Torsion. Urology 72(4): 786-789. 
2. Njeze GE (2012) Testicular torsion: Needless testicular loss can be prevented. Niger J Clin Pract 15(2): 182-185.

3. Cimador M, DiPace MR, Castagnetti M, DeGrazia E (2007) Predictors of testicular viability in testicular torsion. J Pediatr Urol 3(5): 387-390.

4. Figueroa V, Pippi JLS, Braga LH, Romao R, Koyle MA, et al. (2012) Comparative analysis of detorsion alone versus detorsion and tunica albuginea decompression (Fasciotomy) with tunica vaginalis flap coverage in the surgical management of prolonged testicular ischemia. J Urol 188(4 Supl): 1417-1423.

5. Sessions AE, Rabinowitz R, Hulbert WC, Goldstein MM, Mevorach RA, et al. (2003) Testicular torsion: Direction, degree, duration and disinformation. J Urol 169(20): 663-665.

6. Harvey M, Chanwai G, Cave G (2009) Manual testicular detorsion under propofol sedation. Case Rep Med 2009: 1-2.

7. Garel L, Dubois J, Azzie G, Filiatrault D, Grignon A, et al. (2000) Preoperative manual detorsion of the spermatic cord with Doppler ultrasound monitoring in patients with intravaginal acute testicular torsion. Pediatr Radiol 30(1): 41-44.

8. Cornel EB, Karthaus HF (1999) Manual derotation of the twisted spermatic cord. BJU Int 83(6): 672-674.

9. Dunn JP (2008) Manual detorsion of the testicle. NZ Med J 121(1280): 82.

10. Kiesling VJ, Schroeder DE, Pauljev P, Hull J (1984) Spermatic cord block and manual reduction: primary treatment for spermatic cord torsion. J Urol 132(5): 921-923.

11. Mrhac L, Zakko S, Mohammed SJ, Kaya MA (1997) Redistribution. the sign of the successful testicular detorsion. Clin Nucl Med 22(7): 502.

12. Smith RE (1934) Manipulation treatment of trosioin of testicle. Clin J 250-252.
13. Nash SG (1893) Acute torsion of the spermatic cord: reduction, immediate relief. BMJ 1: 743-744.

14. Perry S, Bryant H (1898) Axial Rotation of the testicle (torsion of spermatic cord) causing strangulation. Birmingham Med Rev 13: 279289.

15. Drlík M, Kočvara R (2013) Torsion of spermatic cord in children: A review. J Pediatr Urol 9(3): 259-266.

16. Smith GI (1955) Cellular changes from graded testicular ischemia. J Urol 73(2): 355-362.

17. Thomas WE, Cooper MJ, Crane GA, Lee G, Williamson RC, et al. (1984) Williamson RCN: Testicular exocrine malfunctino after torsion. Lancet 2(8416): 1357-60.

18. Tryfonas G, Violaki A, Tsikopoulos G, Avtzoglou P, Zioutis J, et al. (1994) Late postoperative results in males treated for testicular torsion during childhood. J Pediatr Surg 29(4): 553-556.

19. Brasso K, Andersen L, Kay L, Wille-Jørgensen P, Linnet L, et al. (1993) Testicular torsion: a follow-up study. Scand J Urol Neprhrol 27(1): 1-6.

20. Hadziselimovic F, Geneto R, Emmons LR (1998) Increased apoptosis in the contralateral testes of patients with testicular torsion as a factor for infertility. J Urol 160(3 Pt 2): 1158 - 1160.

21. Turner TT, Brown KJ (1993) Spermatic cord torsion:loss of spermatogenesis despite return blood flow. Biol Reprod 49(2): 401407.

22. Turner TT, Bang HJ, Lysiak JL (2004) The molecular pathology of experimental testicular torsion suggests adjunct therapyu to surgical repair. J Urol 176(6 Pt 2): 2574-2578.

23. Anaya-Prado R, Toledo-Pereyra LH, Lentsch AB, Ward PA (2002) Ischemia/reperfusion injury. J Surg Res 105(2): 248-58.

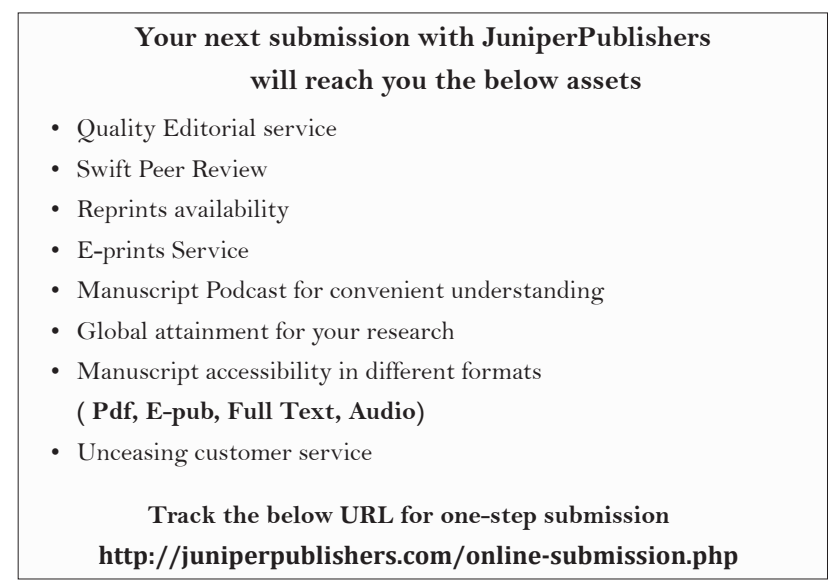

